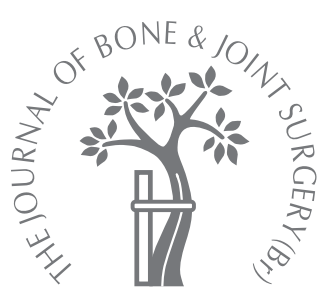

R. W. Brouwer, S. M. A. BiermaZeinstra, A. J. van Koeveringe, J. A. N. Verhaar

From Erasmus Medical Centre, Rotterdam, The Netherlands

\title{
Patellar height and the inclination of the tibial plateau after high tibial osteotomy
}

\author{
THE OPEN VERSUS THE CLOSED-WEDGE TECHNIQUE
}

Our aim was to compare the degree of patellar descent and alteration in angle of the inclination of the tibial plateau in lateral closing-wedge and medial opening-wedge high tibial osteotomy (HTO) in 51 consecutive patients with osteoarthritis of the medial compartment and varus malalignment. Patellar height was measured by the Insall-Salvati (IS) and the Blackburne-Peel (BP) ratios. The tibial inclination was determined by the Moore-Harvey (MH) method. Multivariate linear regression analysis was used to determine the influence of the type of HTO (closing vs opening wedge) on the post-operative patellar height or tibial inclination. The intra- and interobserver variability of these methods was determined before operation and at follow-up at one year.

After an opening-wedge HTO the patellar height was significantly more decreased (mean post-operative difference: IS = 0.15; $95 \%$ confidence interval (CI) 0.06 to $0.23 ; \mathrm{BP}=0.11 ; 95 \%$ Cl 0.05 to 0.18 ) compared with a closing-wedge HTO.

The angle of tibial inclination differed significantly (mean post-operative difference $\mathrm{MH}=-6.40^{\circ} ; 95 \% \mathrm{Cl}-8.74$ to -4.02 ) between the two HTO techniques, increasing after opening-wedge HTO and decreasing after closing-wedge HTO.

There was no clinically-relevant difference in the intra- and interobserver variability of measurements of patellar height either before or after HTO.

High tibial osteotomy (HTO) for the treatment of osteoarthritis of the medial compartment of the knee delays the need for an arthroplasty. ${ }^{1-3}$ However, total knee replacement after HTO presents more technical problems and complications because of scars, valgus alignment, patella baja and the change in tibial inclination. $^{4-6}$

Our aim in this prospective, randomised study was to compare the severity of patellar descent and alteration in the angle of inclination of the tibial plateau in two different techniques of HTO, a medial opening-wedge and a lateral closing-wedge osteotomy. In addition, in the opening-wedge group we compared the use of plaster and no plaster. We also determined the intra- and interobserver variability of two methods of measurement of patellar height; the Insall-Salvati (IS) and the Blackburne-Peel (BP) ratios, and of one method of measurement of tibial inclination, the MooreHarvey (MH) method. ${ }^{7-9}$

\section{Patients and Methods}

Approval of the Ethics Committee of the university medical centre was obtained for the trial and the patients gave their informed consent.
The criteria for inclusion included osteoarthritis of the medial compartment with medial pain and varus malalignment of the mechanical axis measured on long-standing radiographs. The criteria for exclusion were symptomatic osteoarthritis of the lateral compartment, rheumatoid arthritis, a range of movement of less than $100^{\circ}$, lateral collateral ligament laxity of grade 3 , a history of fracture or previous open operation of the lower limb, and flexion contracture.

Between January 2001 and January 2003, 51 consecutive patients were randomised according to a computer-generated procedure using sealed envelopes. Randomisation involved the following procedures: 1) closing-wedge HTO and a cylinder plaster cast for six weeks postoperatively; 2) opening-wedge HTO and no plaster cast post-operatively and 3) openingwedge HTO and a cylinder plaster cast for six weeks post-operatively.

The primary randomisation was between the closing- and opening-wedge techniques, with the intention to create two groups of equal size. In the opening-wedge group there was a second randomisation for treatment after the osteotomy, namely, with or without a 


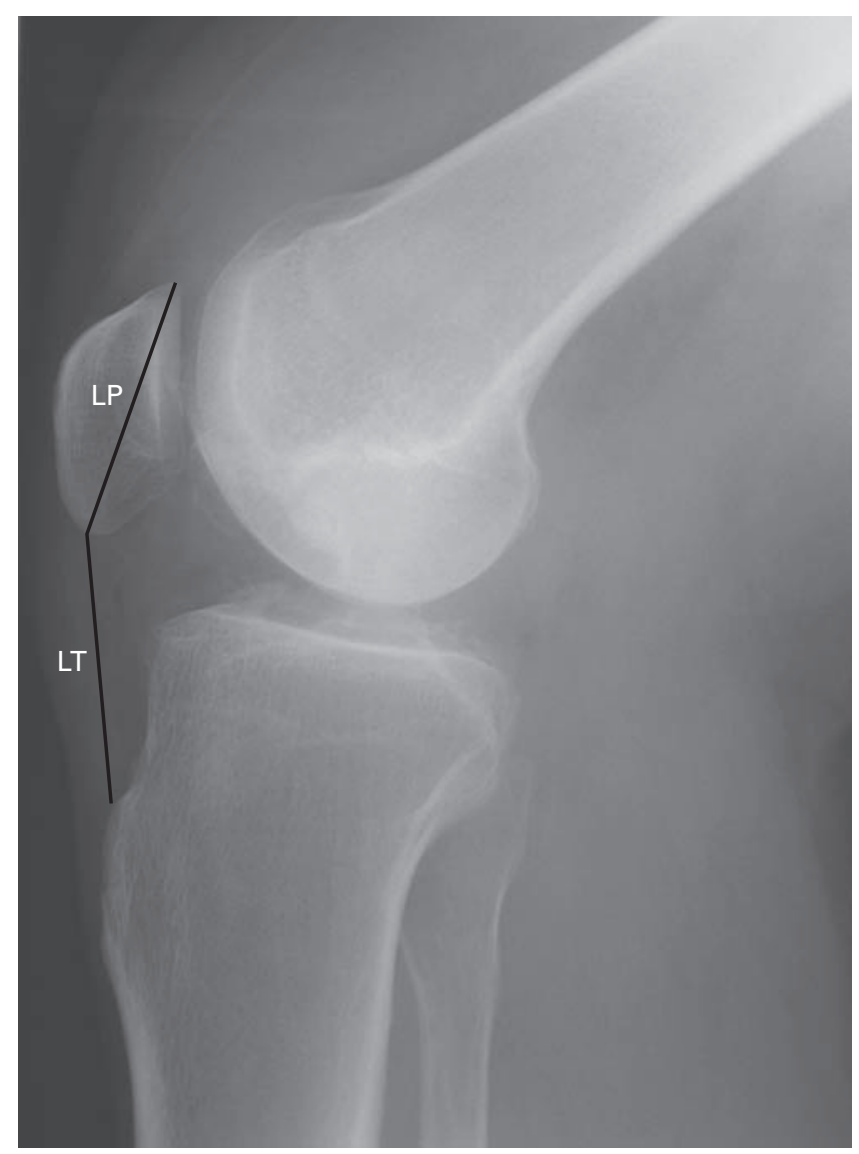

Fig. 1

Radiograph showing determination of the patellar height according to the Insall-Salvati ratio. The length of the patellar tendon (LT) is divided by the longest length of the patella (LP)

plaster cast to determine if a plaster cast influenced the post-operative results.

The goal was to achieve a correction of $4^{\circ}$ in excess of physiological valgus. For the closing-wedge HTO we used the instrumentation of Allopro (Centerpulse, Winterthur, Switzerland). The anterior part of the fibular head (anterior part of the proximal tibiofibular syndesmosis) was resected and the tibial osteotomy was secured with two staples.

The opening-wedge HTO was created by the Puddu HTO (Arthrex, Naples, Florida) instrumentation. The tibial osteotomy was fixed with the Puddu plate. If the opening-wedge was more than $7.5 \mathrm{~mm}$ the void was filled with bone harvested from the ipsilateral iliac crest.

The patients were mobilised on the first post-operative day and partial weight-bearing was allowed in all three groups. After six weeks any plaster cast used was removed.

Standardised radiography was performed pre-operatively and on the first day and 12 months after the operation. Patients who were re-operated on for various reasons during the one-year follow-up period also received standardised radiography before re-operation. In these patients

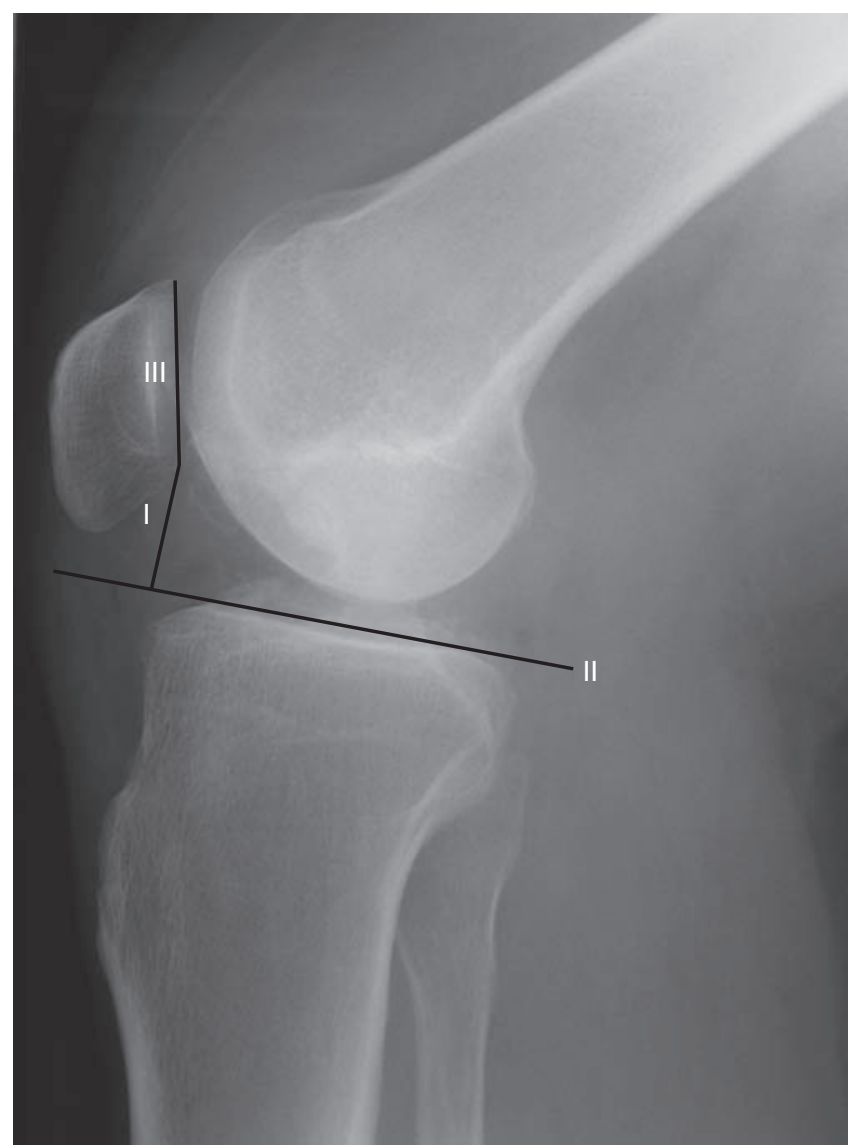

Fig. 2

Radiograph showing determination of the patellar height according to the $\mathrm{BP}$ ratio. The distance (I) from the distal pole of the articular surface of the patella to a perpendicular at the level of the tibia plateau (II) is divided by the length of the patellar articular surface (III).

the measurements just before the re-operation were considered as measurements at the follow-up at one year.

Measurements. Radiography of the knee included a standing posteroanterior view and a true lateral radiograph in at least $30^{\circ}$ of flexion. From all the lateral radiographs the patellar height was measured according to the IS and BP ratios (Figs 1 and 2) and the angle of inclination of the tibial plateau according to the MH method (Fig. 3).

Two observers (RB and AK) measured the patellar height and the angle of inclination of the tibial plateau pre-operatively and follow-up at one year.

Statistical analysis. A multivariate linear regression method was used to analyse the impact of closing- $v$ s openingwedge HTO on changes in patellar height or tibial inclination between measurements made pre-operatively and at the follow-up at one year.

Two multivariate regression analyses were performed. In one the dependent variable was the post-operative patellar height and the independent variables the wedge HTO (opening $v s$ closing) and the pre-operative patellar height. In the second, the dependent variable was the post-opera- 


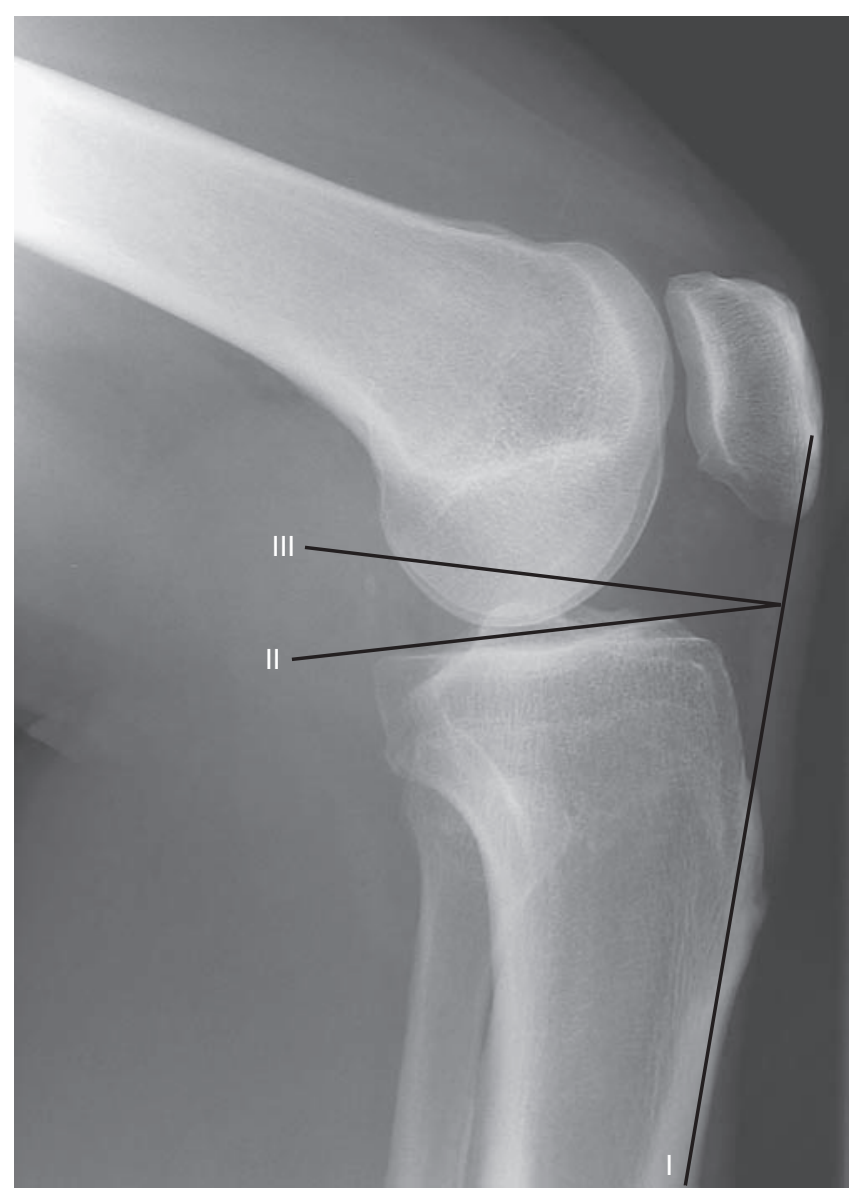

Fig. 3

Radiograph showing the angle of inclination of the tibial plateau according to the $\mathrm{MH}$ method using three lines. The first (I) is tangential to the tibial crest, the second (II) is tangential to the proximal tibial articular surface and the third (III) line is perpendicular to the line of the tibial crest. The angle formed by the second and the third lines is equivalent to the posteroinferior slope of the plateau.

tive tibial inclination and the independent variables the wedge HTO (opening $v s$ closing) and the pre-operative tibial inclination.

Age, gender, the pre-operative hip-knee-ankle angle and the pre-operative degree of osteoarthritis were considered as possible confounders and were included in the model only if they changed the relationship between the dependent variable and the type of HTO by at least $10 \%$. The same was done for the relationship between the above-mentioned dependent variables and the type of post-operative treatment (cast $v s$ no cast) in the group with opening-wedge HTO.

The Pearson correlation between the difference in patellar height (pre-operative minus post-operative) and that of the angle of inclination (pre-operative minus post-operative) was assessed in order to estimate dependency between the outcome measures.

The intra- and interobserver variabilities were expressed as intraclass correlation coefficients (ICC) which vary from
Table I. Details of the patients

\begin{tabular}{|c|c|c|c|c|c|c|}
\hline \multirow[b]{2}{*}{ Male:female } & \multicolumn{2}{|c|}{$\begin{array}{l}\text { Closing-wedge } \\
\text { HTO }^{*}(n=24)\end{array}$} & \multicolumn{2}{|c|}{$\begin{array}{l}\text { Opening-wedge } \\
\text { HTO }(n=26)\end{array}$} & \multicolumn{2}{|c|}{$\begin{array}{l}\text { Total } \\
(n=50)\end{array}$} \\
\hline & $12: 12$ & & $20: 6$ & & $32: 18$ & \\
\hline Mean age in yrs (SD) & 52.6 & (8.5) & 47.7 & $(7.4)$ & 50.1 & (8.2) \\
\hline Mean HKA angle $\left({ }^{\circ}\right)(\mathrm{SD})$ & 187.8 & $(2.6)$ & 185.4 & $(2.7)$ & 186.6 & (2.9) \\
\hline \multicolumn{7}{|c|}{$\begin{array}{l}\text { Ahlbäck score }{ }^{18} \text { medial } \\
\text { compartment }\end{array}$} \\
\hline No osteoarthritis & 1 & & 0 & & 1 & \\
\hline Joint-space narrowing & 22 & & 21 & & 43 & \\
\hline Obliteration & 1 & & 5 & & 6 & \\
\hline \multicolumn{7}{|l|}{ Lateral compartment } \\
\hline No osteoarthritis & 22 & & 23 & & 45 & \\
\hline Joint-space narrowing & 92 & & 3 & & 5 & \\
\hline Obliteration & 0 & & 0 & & 0 & \\
\hline
\end{tabular}

* HTO, high tibial osteotomy

zero (no agreement at all) to 1 (total agreement). Additionally, the approach of Bland and Altman ${ }^{10}$ was used to determine the limits of agreement and to ensure that the repeatability was constant. A p value of 0.05 was considered to be statistically significant.

\section{Results}

One patient was lost to follow-up leaving 18 women and 32 men in the study. Their mean age was 50.1 (SD 8.2) years. The mean pre-operative HKA angle was $186.6^{\circ}$ (SD 2.9) (Table I). In all patients the cruciate ligaments were intact.

Pre-operatively, the mean IS ratio and the mean BP ratios were 0.90 (SD 0.17) and 0.76 (SD 0.11), respectively. The mean angle of inclination of the tibial plateau was $9.6^{\circ}$ (SD 3.1).

A total of 24 patients had a closing-wedge and 26 an opening-wedge HTO; 12 of the opening-wedge group had a plaster cast and the remainder did not.

Four patients required operation during the one-year follow-up period. In one patient in the closing-wedge group this was because of overcorrection (varus HTO) and in another for progression of symptoms (total knee arthroplasty). In the opening-wedge group two patients required re-operation because of recurrent varus alignment (revalgus $\mathrm{HTO}$ ).

Post-operatively, the patellar height according to the IS ratio was 0.92 (SD 0.17) in the closing-wedge group and 0.81 (SD 0.20) in the opening-wedge HTO group. The mean patellar height according to the BP ratio was 0.77 (SD 0.12) in the closing-wedge group and 0.70 (SD 0.16) in the opening-wedge group (Table II; Fig. 4).

The z-residuals plotted against the predicted residuals gave a normal distributed scatterplot for all models presented, meaning that these values were independent of each other, and that this specific condition for executing linear regression was satisfied.

Testing for possible confounding between the type of HTO and the post-operative patellar height or post-operative angle of inclination showed that the pre-operative hipknee-ankle angle was a confounder. Adjustment for this 
Table II. Mean (SD) patellar height according to the Insall-Salvati (IS) and Blackburne-Peel (BP) ratios and the mean (SD) inclination of the tibial plateau according to the Moore-Harvey $(\mathrm{MH})$ method after closing- and opening-wedge high tibial osteotomy (HTO)

\begin{tabular}{|c|c|c|c|c|}
\hline & $\begin{array}{l}\text { Closing-wedge } \\
(n=24)\end{array}$ & $\begin{array}{l}\text { Opening-wedge } \\
(\mathrm{n}=26)\end{array}$ & $\begin{array}{l}\text { Adjusted* post-operative } \\
\text { difference, } \beta(95 \% \mathrm{Cl})\end{array}$ & $\begin{array}{l}\text { p value for adjusted } \\
\text { post-operative difference }\end{array}$ \\
\hline \multicolumn{5}{|l|}{ Patellar height } \\
\hline \multicolumn{5}{|l|}{ IS ratio } \\
\hline Pre-operative & $0.91(0.18)$ & $0.90(0.17)$ & & \\
\hline Post-operative & $0.92(0.17)$ & $0.81(0.20)$ & $0.15(0.06 ; 0.23)$ & 0.001 \\
\hline \multicolumn{5}{|l|}{ BP ratio } \\
\hline Pre-operative & $0.75(0.10)$ & $0.78(0.11)$ & & \\
\hline Post-operative & $0.77 \quad(0.12)$ & $0.70(0.16)$ & $0.11(0.05 ; 0.18)$ & 0.001 \\
\hline \multicolumn{5}{|l|}{ Tibial slope } \\
\hline \multicolumn{5}{|l|}{$\mathrm{MH}\left({ }^{\circ}\right)$} \\
\hline Pre-operative & $9.79(2.70)$ & $9.50(3.50)$ & & \\
\hline Post-operative & $6.02(3.87)$ & $11.87(4.89)$ & $-6.40(-8.74 ;-4.02)$ & $<0.001$ \\
\hline
\end{tabular}

* this table reflects the post-operative difference between the closing- and opening-wedge HTO, adjusted for baseline measurement of patellar height or tibial slope, and hip-knee-ankle angle at baseline, estimated in linear regression analysis. The closingwedge HTO is compared with the opening-wedge HTO meaning that a positive value indicates more patella height or more slope for the closing HTO, while a negative value indicates the opposite; Cl, confidence interval.

value in multivariate linear regression (type of HTO and pre-operative hip-knee-ankle angle and pre-operative patellar height as independent variables; post-operative patellar height as dependent variable) showed that the post-operative mean difference in patellar height due to the type of HTO was 0.15 ( $p=0.001 ; 95 \%$ CI 0.06 to 0.23 ) for the IS ratio and 0.11 ( $\mathrm{p}=0.001 ; 95 \%$ CI 0.05 to 0.18$)$ for the $\mathrm{BP}$ ratio.

The mean angle of inclination of the tibial plateau was $11.9^{\circ}$ (SD 4.9) in the opening-wedge HTO group and $6.0^{\circ}$ (SD 3.9) in the closing-wedge HTO group (Table II). The adjusted mean post-operative difference was $-6.40^{\circ}$ (p < 0.001; 95\% CI -8.74 to -4.02 ; Fig. 5).

In the opening-wedge HTO group, the subgroup without plaster $(\mathrm{n}=14)$ had mean post-operative IS and BP ratios of 0.79 (SD 0.18) and 0.66 (SD 0.12), respectively, compared with 0.83 (SD 0.24) and 0.75 (SD 0.20), respectively, in the subgroup with plaster $(\mathrm{n}=12)$. Multivariate linear regression analysis (plaster $v s$ no plaster, pre-operative hip-kneeankle angle, and pre-operative patellar height as independent variables; post-operative patellar height as a dependent variable) showed no statistically significant difference between groups with and without plaster. The mean postoperative difference because of post-operative treatment was 0.012 ( $\mathrm{p}=0.83 ; 95 \% \mathrm{CI}-0.10$ to 0.13$)$ for the IS and 0.07 for the BP ratios ( $p=0.15 ; 95 \%$ CI -0.03 to 0.16 ).

After HTO there was a significant correlation between the differences in patellar height and the angle of inclination of the tibial plateau, for the patellar height measured both by the IS $(r=0.398 ; \mathrm{p}=0.005)$ and the BP $(r=0.453$; $\mathrm{p}=0.001)$ methods.

The ICCs of the IS and BP ratios together with the $\mathrm{MH}$ slope estimations are presented in Table III. In all measurements the ICCs were reasonable. For the two ratios the intra-observer agreement was superior to the interobserver agreement in every situation. For the $\mathrm{MH}$ assessments intra- and interobserver agreement was similar throughout.

For measurements of patellar height the Bland-Altman approach showed that the plot of the difference in the mea-

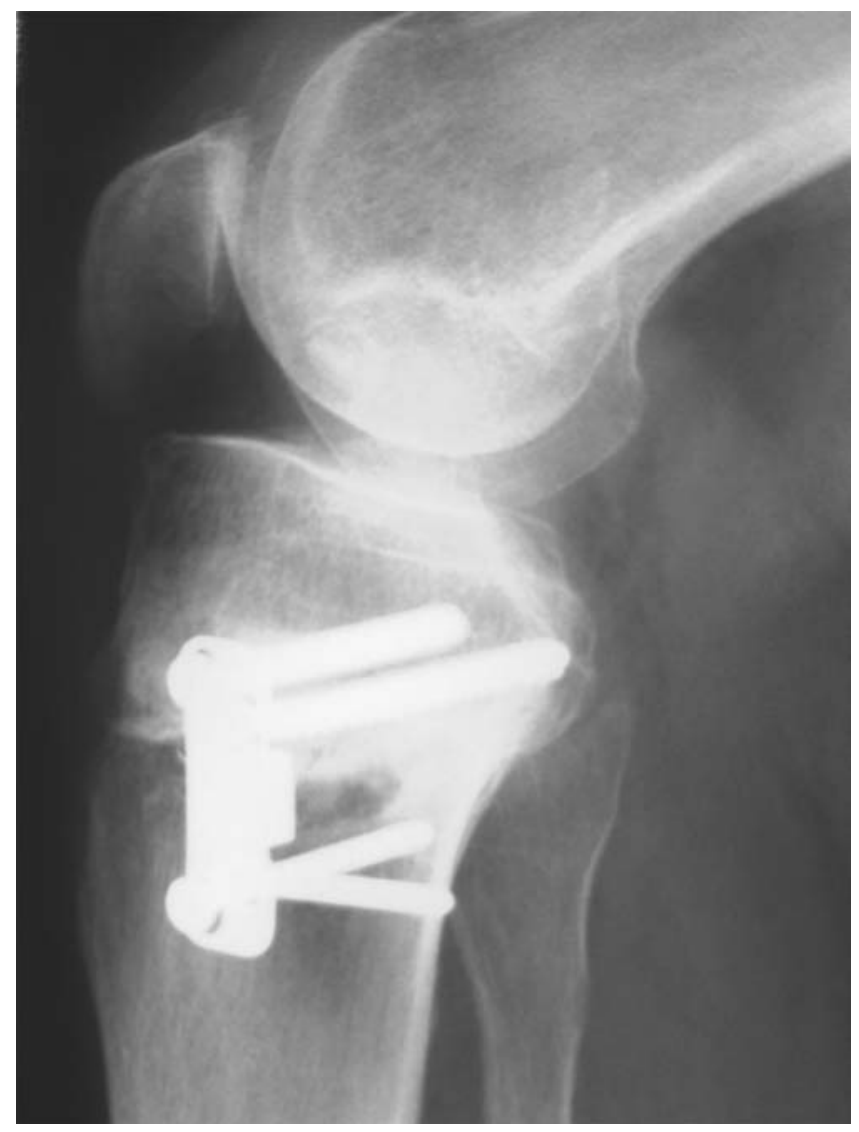

Fig. 4

Radiograph showing patella baja after an opening-wedge high tibial osteotomy.

surements recorded by the two observers for each patient against their mean indicated that the differences were not related to the size of the measurement. However, for measurements of the angle of inclination of the tibial plateau the Bland-Altman plot showed that the differences increased when the size of the measurements became larger. 
Table III. Intravenous correlation coefficient (ICC) of the Insall-Salvati (IS) and Blackburne-Peel (BP) ratios pre-operatively and after closing-wedge high tibial osteotomy (HTO)

\begin{tabular}{lllllll}
\hline & \multicolumn{2}{l}{ IS ICC } & \multicolumn{3}{c}{ BP ICC } & \multicolumn{2}{c}{ MH* ICC } \\
\cline { 2 - 7 } & Intra & Inter & Intra & Inter & Intra & Inter \\
\hline Pre-operative & 0.83 & 0.45 & 0.81 & 0.42 & 0.65 & 0.73 \\
Post-operative & 0.86 & 0.63 & 0.79 & 0.56 & 0.82 & 0.82 \\
Closing-wedge HTO & 0.87 & 0.56 & 0.79 & 0.79 & 0.71 & 0.77 \\
Opening-wedge HTO & 0.85 & 0.66 & 0.78 & 0.45 & 0.78 & 0.82 \\
\hline * & & & & & &
\end{tabular}

The post-operative mean differences between the two observers and the limits for agreement were as follows: IS: $-0.20(-0.56$ to 0.17$)$, BP: $-0.02(-0.32$ to 0.30$)$ and $\mathrm{MH}$ : $1.80^{\circ}$ (-4.69 to 8.29$)$. These limits of agreement were comparable with the pre-operative limits of agreement.

\section{Discussion}

Patella baja, the change in tibial slope and valgus alignment after HTO can all cause technical difficulties during total knee replacement particularly in relation to eversion of the patella, exposure of the lateral compartment, and placing the tibial component in the correct position, both for rotation and slope.

In our study more patella baja was created after an opening-wedge HTO and the angle of inclination increased. After a closing-wedge HTO the angle of inclination decreased.

Pre-operatively, the hip-knee-ankle angles differed between the closing- and opening-wedge groups. This angle proved to be a confounder for the relationship between post-operative patellar height or the post-operative angle of inclination of the tibial plateau and the type of HTO. Correction for this confounder resulted in an even stronger difference in outcome between the opening- and closingwedge HTO groups.

For measurement of patellar height we chose the IS method because it is most frequently used. Secondly, we applied the BP method in accordance with the recommendation of Seil et $\mathrm{al}^{11}$ following their comparison of different methods of measurements of patellar height. In their study on 22 non-operated knees the BP method showed the lowest interobserver variability and best discrimination of patella alta, norma and baja. ${ }^{11}$

In our study there was no clinically relevant difference in the intra- and interobserver variability of measurements of patellar height either before or after operation.

According to both the IS ratio and the BP method, there was significantly more patellar descent after an openingwedge HTO. However, we agree with Kaper et $\mathrm{al}^{5}$ that the $\mathrm{BP}$ ratio is not a valid measurement for patellar height after

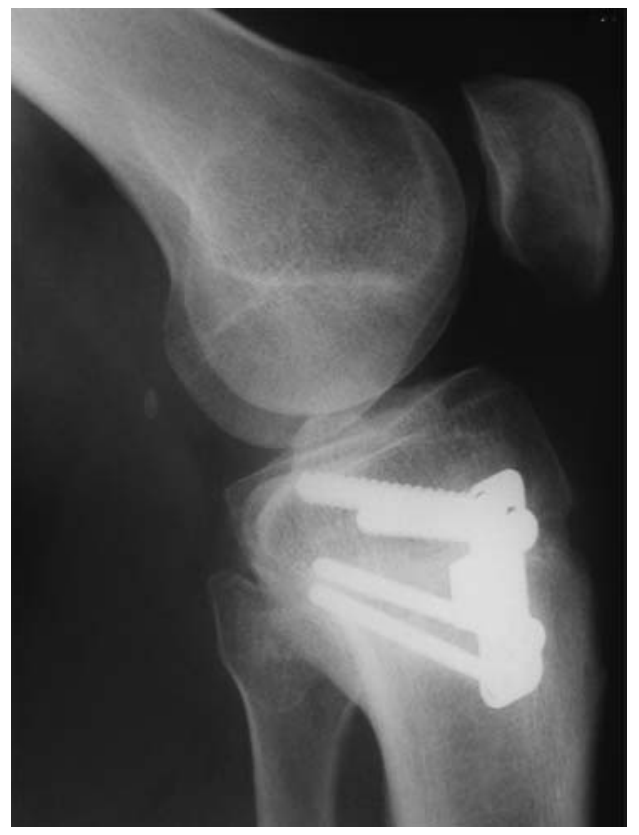

Fig. $5 a$

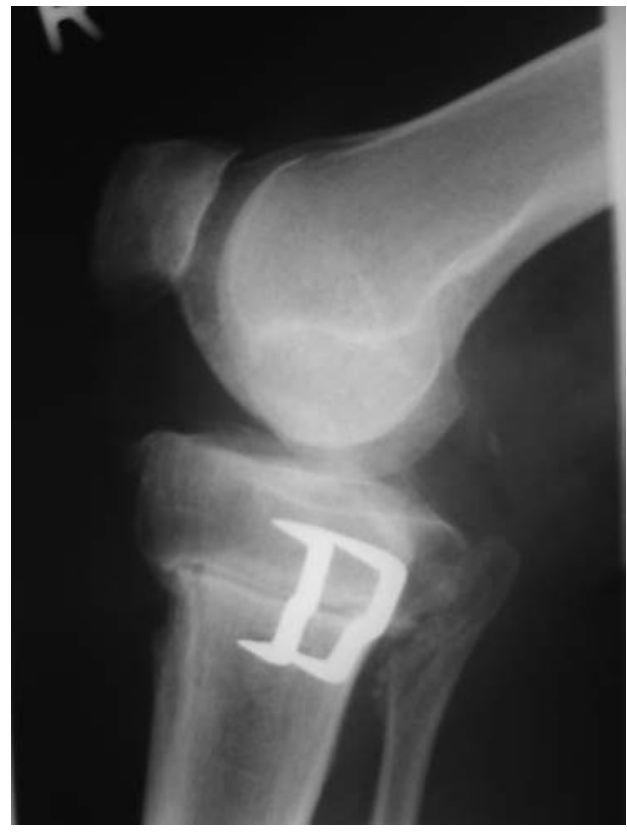

Fig. $5 b$

Radiographs showing a) the increase of the inclination of the angle of the tibial plateau after an opening-wedge high tibial osteotomy (HTO) and b) decrease of the inclination angle of the tibial plateau after a closing-wedge HTO. 
HTO, because a change in the angle of inclination of the tibial plateau adversely affects the reproducibility of this measurement. In the retrospective trial of Tigani et $\mathrm{al}^{12}$ more patellar descent occurred after opening-wedge compared with closing-wedge HTO. However, they used the Caton-Dechamps method which is thought to be insufficient for measurements of the patellar height after HTO or total knee arthroplasty because this ratio is adversely affected by the joint line. ${ }^{13}$

According to Kaper et $\mathrm{al}^{5}$ loss of tibial slope after closing-wedge HTO was significantly associated with patellar baja. Our results showed that an increase in the tibial slope was also significantly associated with patellar baja according to the IS and BP ratios. Patellar descent was caused by several factors such as scarring in and around the patellar ligament because of immobilisation, the formation of new bone at the site of the osteotomy, the alteration of tibial inclination and the elevation of the tibial plateau after opening-wedge osteotomy. ${ }^{5,12,14-16}$

In a prospective, randomised trial, Nakamura et $\mathrm{al}^{17}$ showed that an opening-wedge osteotomy by hemicallotasis caused little change in the length of the patellar tendon or angle of inclination of the tibial plateau while both decreased in a group which received a dome osteotomy. Hemicallotasis caused less change in patellar length and angle of inclination of the tibial slope because the osteotomy was below the insertion of the patellar tendon and the external fixator could be adjusted after osteotomy.

HTO with rigid fixation and early movement is advised to avoid patella baja. ${ }^{14,15}$ However, in spite of immobilisation, we did not have significant patellar descent after a closing-wedge HTO. After an opening-wedge HTO, the degree of patellar descent did not differ significantly between the plaster and non-plaster subgroups.

A change in inclination after HTO may have several causes. The first is the precision of both opening- and closing-wedge HTOs which should be parallel to the angle of inclination of the tibial plateau. Secondly, in openingwedge HTO, if the plate is placed too anteriorly more inclination will be created.

Caution with regard to the neurovascular bundle can cause incomplete bony resection posteriorly during removal of the wedge and can cause loss of inclination after closing-wedge HTO. Retained posterolateral support from the proximal tibiofibular syndesmosis is another possible reason for loss of inclination of the tibial slope after closing-wedge HTO. ${ }^{5}$

Loss of inclination after closing-wedge HTO produces a relative elevation of the posterior cruciate ligament. ${ }^{5}$ When planning a total knee arthroplasty after HTO it should be borne in mind that the patellar height and angle of inclination of the tibial plateau may have been altered.

No benefits in any form have been received or will be received from a commercial party related directly or indirectly to the subject of this article.

\section{References}

1. Aglietti P, Buzzi R, Vena LM, Baldini A, Mondaini A. High tibial osteotomy for medial gonarthrosis: a 10- to 12-year study. J Knee Surg 2003;16:21-6.

2. Coventry MB, Ilstrup DM, Wallrichs SL. Proximal tibial osteotomy: a clinical longterm study of 87 cases. J Bone Joint Surg [Am] 1993;75-A:196-201.

3. Sprenger TR, Doerzbacher JF. Tibial osteotomy for the treatment of varus gonarthrosis: survival and failure analysis to twenty-two years. J Bone Joint Surg [Am] 2003;85-A:469-74.

4. Haddad FS, Bentley G. Total knee arthroplasty after high tibial osteotomy: a medium-term review. J Arthroplasty 2000;15:597-603.

5. Kaper BP, Bourne RB, Rorabeck CH, Macdonald SJ. Patellar infera after high tibial osteotomy. J Arthroplasty 2001;16:168-73.

6. Katz MM, Hungerford DS, Krackow KA, Lennox DW. Results of total knee arthroplasty after failed proximal tibial osteotomy for osteoarthritis. J Bone Joint Surg [Am] 1987;69-A:225-33.

7. Insall J, Salvati E. Patella position in the normal knee joint. Radiology 1971;101:101-4.

8. Blackburne JS, Peel TE. A new method of measuring patellar height. J Bone Joint Surg [Br] 1977;59-B:241-2.

9. Moore TM, Harvey JP Jr. Roentgenographic measurement of tibial plateau depression due to fracture. J Bone Joint Surg [Am] 1974;56-A:155-60.

10. Bland JM, Altman DG. Statistical methods for assessing agreement between the two methods of clinical measurement. Lancet 1986;1:307-10.

11. Seil R, Müller B, Georg T, Kohn D, Rupp S. Reliability and interobserver variability in radiological patellar height ratios. Knee Surg Sports Traumatol Arthrosc 2000;8: 231-6.

12. Tigani D, Ferrari D, Trentani P, Barbanti-Broadano G, Trentani F. Patellar height after high tibial osteotomy. Int Orthop 2001;24:331-4.

13. Grelsamer RP. Patella baja after total knee arthroplasty: is it really patella baja? J Arthroplasty 2002;17:66-9.

14. Billings A, Scott DF, Camargo MP, Hofmann AA. High tibial osteotomy with a calibrated osteotomy guide, rigid internal fixation, and early motion: long-term followup. J Bone Joint Surg [Am] 2000;82-A:70-9.

15. Closkey RF, Windsor RE. Alteration in the patella after a high tibial or distal femora osteotomy. Clin Orthop 2001;389:51-6.

16. Scuderi GR, Windsor RE, Insall JN. Observation on patellar height after proximal tibial osteotomy. J Bone Joint Surg [Am] 1989;71-A:245-8.

17. Nakamura E, Mizuta H, Kudo S, Takagi K, Sakamoto K. Open-wedge osteotomy of the proximal tibia with hemicallotasis. J Bone Joint Surg [Br] 2001;83-B:1111-14.

18. Ahlbäck S. Osteoarthrosis of the knee: a radiograph investigation. Acta Radiol Diagn (Stockh) 1968;Suppl 277:7-72. 